\title{
Seroprevalence of Baylisascaris procyonis Infection among Humans, Santa Barbara County, California, USA, 2014-2016
}

\author{
Sara B. Weinstein, Camille M. Lake, ${ }^{1}$ \\ Holly M. Chastain, David Fisk, Sukwan Handali, \\ Philip L. Kahn, Susan P. Montgomery, \\ Patricia P. Wilkins, Armand M. Kuris, \\ Kevin D. Lafferty
}

Baylisascaris procyonis (raccoon roundworm) infection is common in raccoons and can cause devastating pathology in other animals, including humans. Limited information is available on the frequency of asymptomatic human infection. We tested 150 adults from California, USA, for $B$. procyonis antibodies; 11 were seropositive, suggesting that subclinical infection does occur.

$\mathrm{T}_{\mathrm{a}}^{\mathrm{h}}$ he raccoon roundworm (Baylisascaris procyonis) is a potential health risk to humans. Infected raccoons release eggs in their feces, and these eggs accumulate at communal defecation sites (latrines). When nonraccoon hosts consume eggs, larva migrans can cause blindness and fatal neurologic sequelae $(1,2)$. Less than $5 \%$ of migrating larvae reach the brain, and experimental studies suggest that host size, infection site, and inoculating dose drive pathology (1).

Reported human disease cases are rare; however, there is growing evidence for more frequent asymptomatic infections. For example, a recent study showed that $7 \%$ of wildlife rehabilitators had Baylisascaris-specific antibodies (3). Large and heavily infected raccoon populations probably contaminate many regions with $B$. procyonis eggs (1). These microscopic eggs can survive for years (4), so anyone living in regions with infected raccoons probably has an exposure risk.

Santa Barbara County, California, USA, is a potential high-risk area for exposure to $B$. procyonis eggs. In

Author affiliations: University of California, Santa Barbara,

California, USA (S.B. Weinstein, C.M. Lake, A.M. Kuris,

K.D. Lafferty); IRHC Inc., Atlanta, Georgia, USA (H.M. Chastain);

Centers for Disease Control and Prevention, Atlanta,

(H.M. Chastain, S. Handali, S.P. Montgomery, P.P. Wilkins);

Sansum Clinic, Santa Barbara (D. Fisk); University of California, Berkeley, California, USA (P.L. Kahn); US Geological Survey

Western Ecological Research Center, Santa Barbara

(K.D. Lafferty)

DOI: https://doi.org/10.3201/eid2308.170222
Santa Barbara County, a baylisascariasis case was reported in a toddler in $2002(1,5)$; raccoon roundworm consistently infects $>80 \%$ of raccoons $(6,7)$; and latrines are abundant in residential areas (J.F. Mendez, University of California, pers. comm., 2017 Feb 1), potentially exposing residents to roundworms. We describe the use of a parasite-specific antibody assay to detect subclinical B. procyonis infections in adult residents of Santa Barbara County. Recruitment, enrollment, and sampling methods were reviewed and approved by the Santa Barbara Cottage Hospital and University of California institutional review boards (14-06u).

\section{The Study}

During 2014-2016, we provided public education about raccoon roundworm infection and offered free testing to healthy adults (18-75 years of age) who had lived in Santa Barbara County for $\geq 3$ years. We recruited participants by word of mouth and flyers and through presentations at public outreach events and classes at the local university, natural history museum, zoo, and other venues. We also provided information about testing to local wildlife rehabilitators and researchers working with raccoons and B. procyonis.

We collected serum samples from a convenience sample of 150 volunteers. This sampling included serum from 5 wildlife rehabilitators and 7 researchers; however, we considered results from these 12 persons separately because their $B$. procyonis roundworm exposure was expected to be higher than that for the general population.

We collected $\approx 5 \mathrm{~mL}$ of blood from each volunteer and allowed the samples to clot. We then centrifuged the samples at $1,500 \times g$ for $15 \mathrm{~min}$, separated them, and stored them at $-80^{\circ} \mathrm{C}$. Participants $(149 / 150)$ filled out a questionnaire on demographic characteristics and potential risk factors, such as pet ownership, pet and wildlife feeding practices, past contact with raccoons or raccoon feces, and frequency of raccoon observations around their neighborhood and residence (online Technical Appendix, https://wwwnc.cdc.gov/EID/article/23/8/17-0222Techapp1.pdf). We deidentified samples and tested them for $B$. procyonis $\mathrm{IgG}$ by using a recombinant $B$. procyonis repeat antigen 1 protein Western blot assay, which has ${ }^{1}$ Current affiliation: Uniformed Services University, Bethesda, Maryland, USA. 
$88 \%$ sensitivity and $98 \%$ specificity $(3,8)$. We generated prevalence estimates by using EpiTools (9), calculating 95\% confidence limits (CLs) for an imperfect diagnostic assay (10). We then compared questionnaire responses from seropositive participants with those from seronegative participants by using exact binomial tests in $\mathrm{R}$ (11).

The 12 researchers and wildlife rehabilitators tested negative for B. procyonis antibodies. Among the remaining 138 volunteers, 11 tested positive (apparent prevalence 8.0\%, Wilson CL 4.5\%-13.7\%; adjusted prevalence 6.9\%, accounting for test sensitivity and specificity; Blaker exact CL 2.5\%-13.4\%). All 11 B. procyonis-seropositive participants had seen raccoons in their neighborhood during the past year, and 7 had seen 1 in their yard during the past month. Of the $11 \mathrm{~B}$. procyonis-positive study participants, 9 reported no contact with raccoons or their feces; for the 2 who reported contact, the possible exposures occurred 2 and 12 months before testing. Persons with positive serologic test results ranged in age from 20 to 72 years and included an engineer, student, administrator, researcher, social worker, zoo volunteer, and retiree. Some seropositive persons owned dogs, fed animals outside, gardened, and had sandboxes. However, persons with negative serologic test results gave similar responses ( $p>0.15$ for all comparisons), and we found too few infected persons to identify risk factors (Table).

\section{Conclusions}

We estimate that $\approx 7 \%$ of the sampled persons had antibodies to raccoon roundworm; however, this convenience sample does not represent all county residents. Our recruiting strategy probably introduced income, age, and education biases, and, because we recruited study participants during presentations to groups interested in wildlife and outdoor activities, we might have selected a sample population with a greater exposure risk. Furthermore, because participants could receive their test results, we expect that persons concerned about past exposure were more likely to participate. Although most baylisascariasis is attributed to $B$. procyonis, cross-reactivity between the $B$. procyonis recombinant antigen assay and other less common Baylisascaris spp. is not well characterized and warrants further study. This survey suggests that subclinical Baylisascaris infection occurs in the general population; however, additional studies would improve prevalence estimates. These surveys could also include children because most clinical $B$. procyonis infections occur in persons $<2$ years of age $(1,2)$ and it is unclear how long antibodies remain after exposure.

Despite frequent contact with raccoons and their feces, no sampled wildlife rehabilitators or researchers tested positive for B. procyonis antibodies. Most wildlife rehabilitators and all researchers examined were aware of $B$. procyonis and took precautions when handling raccoons, feces, and parasites. Although the finding of no infection in these high-risk groups could be a reflection of small sample size, it does suggest that preventive measures are effective.

Subclinical human Baylisascaris infections might occur wherever humans and infected raccoons overlap. These infections are likely more widespread than previously assumed, and their health risk remains an open question. Subclinical infection might result from lower intensity infection or depend on which tissues are infected (1). Lowintensity infection in organs, such as the brain, could result in subtle clinical manifestations, and clinical and serologic evidence is required to understand the full public health effect of Baylisascaris infections.

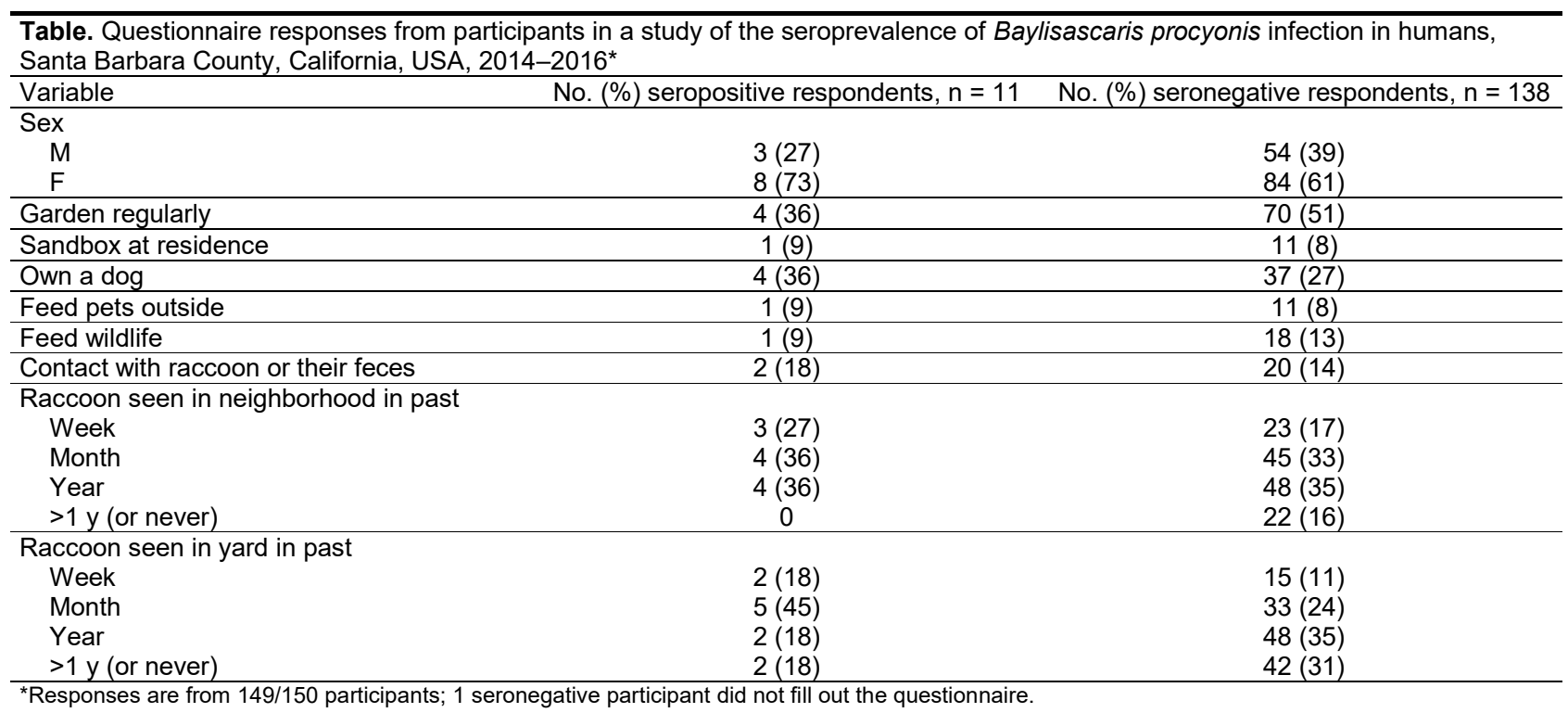




\section{Acknowledgments}

We thank all volunteers who provided samples, Pacific Diagnostic Laboratories and Cottage Health for

facilitating sampling, Ana Elisa Garcia-Vedrenne for Spanish translations, and everyone who wore the raccoon suit during outreach events.

Financial support was provided by the National Science Foundation Graduate Research Fellowships program (grant no. 1144085 to S.B.W.).

Dr. Weinstein recently completed her PhD dissertation in the Department of Ecology, Evolution and Marine Biology at the University of California, Santa Barbara. Her research interests include parasite ecology and evolution.

\section{References}

1. Kazacos KR. Baylisascaris larva migrans: US Geological Survey circular 1412. Reston (VA): US Geological Survey; 2016.

2. Graeff-Teixeira C, Morassutti AL, Kazacos KR. Update on baylisascariasis, a highly pathogenic zoonotic infection. Clin Microbiol Rev. 2016;29:375-99. http://dx.doi.org/10.1128/ CMR.00044-15

3. Sapp SGH, Rascoe LN, Wilkins PP, Handali S, Gray EB, Eberhard M, et al. Baylisascaris procyonis roundworm seroprevalence among wildlife rehabilitators, United States and Canada, 2012-2015. Emerg Infect Dis. 2016;22:2128-31. http://dx.doi.org/10.3201/eid2212.160467

4. Shafir SC, Sorvillo FJ, Sorvillo T, Eberhard ML. Viability of Baylisascaris procyonis eggs. Emerg Infect Dis. 2011;17:1293-5. http://dx.doi.org/10.3201/eid1707.101774

5. Schultz T. "Raccoon roundworm" infection confirmed. Santa Barbara News-Press. 2002;B1:3.

6. Weinstein SB. Baylisascaris procyonis demography and egg production in a California raccoon population. J Parasitol. 2016;102:622-8. http://dx.doi.org/10.1645/15-747

7. Moore L, Ash L, Sorvillo F, Berlin OGW. Baylisascaris procyonis in California. Emerg Infect Dis. 2004;10:1693-4. http://dx.doi.org/ 10.3201/eid1009.040034

8. Rascoe LN, Santamaria C, Handali S, Dangoudoubiyam S, Kazacos KR, Wilkins PP, et al. Interlaboratory optimization and evaluation of a serological assay for diagnosis of human baylisascariasis. Clin Vaccine Immunol. 2013;20:1758-63. http://dx.doi.org/10.1128/CVI.00387-13

9. Sergeant ESG. EpiTools epidemiological calculators. Canberra (Australia): Ausvet Pty Ltd; 2016 [cited 2016 Dec 5]. http://epitools.ausvet.com.au

10. Reiczigel J, Földi J, Ozsvári L. Exact confidence limits for prevalence of a disease with an imperfect diagnostic test. Epidemiol Infect. 2010;138:1674-8. http://dx.doi.org/10.1017/ S0950268810000385

11. R Core Team. R: A language and environment for statistical computing. 3.3.0 ed. Vienna: R Foundation for Statistical Computing; 2016.

Address for correspondence: Sara B. Weinstein, Ecology, Evolution and Marine Biology, University of California, Santa Barbara, CA 93106-9620, USA; email: Batrachoseps@gmail.com

\section{EID Podcast: Deadly Parasite in Raccoons}

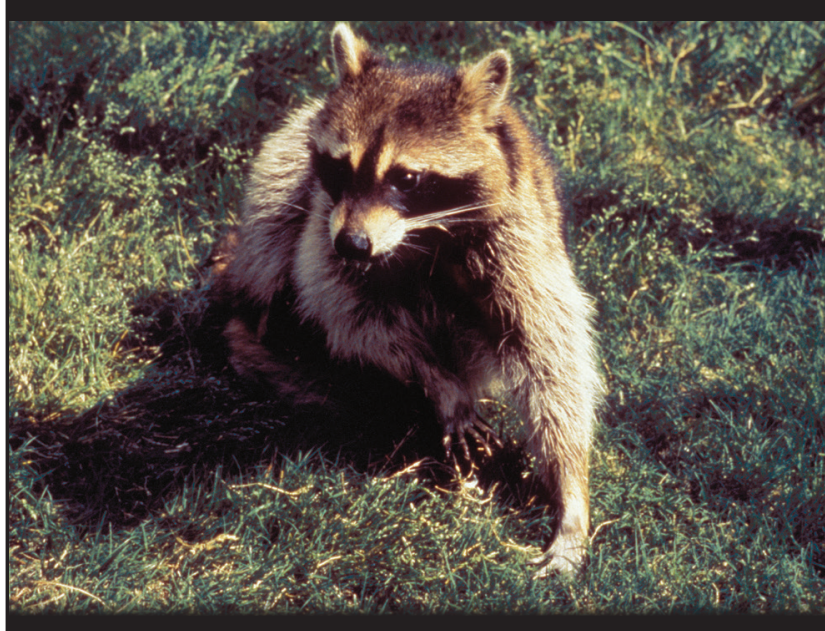

Infection with Baylisascaris procyonis roundworms is rare but often fatal and typically affects children. $B$. procyonis, the common intestinal roundworm of raccoons, has increasingly been recognized as a source of severe, often fatal, neurologic disease in humans, particularly children. Although this devastating disease is rare, lack of effective treatment and the widespread distribution of raccoons in close association with humans make baylisascariasis a disease that seriously affects public health. Raccoons infected with $B$. procyonis roundworms can shed millions of eggs in their feces daily. Given the habit of raccoons to defecate in and around houses, information about optimal methods to inactivate $B$. procyonis eggs is critical for the control of this disease. However, little information is available about survival of eggs and effective disinfection techniques. Additional data provide infomation on thermal death point and determining the impact of desiccation and freezing on the viability of $B$. procyonis eggs to provide additional information for risk assessments of contamination and guide attempts at environmental decontamination.

\section{Visit our website to listen:}

https://www2c.cdc.gov/podcasts/ player.asp?f $=\mathbf{8 6 2 0 6 7 5}$

EMERCING

INFECTIOUS DISEASES 